\title{
Evaluación fibroscópica de la deglución
}

\section{Fibroscopic evaluation of swallowing}

Gonzalo Nazar $\mathrm{M}^{1}$, Andrés Ortega $\mathrm{T}^{1}$, Andrés Godoy $\mathrm{M}^{2}$, José Miguel Godoy $\mathrm{M}^{3}$, Inés Fuentealba $\mathrm{M}^{4}$.

\section{RESUMEN}

Introducción: La disfagia orofaríngea es una condición patológica frecuente, especialmente en pacientes añosos. Se asocia a una serie de enfermedades (principalmente neurológicas y traumáticas), y conlleva un riesgo considerable de aspiración y neumonía La evaluación fibroscópica de la deglución es una técnica que permite estudiar la fisiología de la deglución, estimar el riesgo de aspiración y orientar sobre la forma más segura de alimentar al paciente.

Objetivo: Revisar la experiencia de nuestra institución en la evaluación fibroscópica de la deglución.

Material y método: Entre abril de 2006 y julio de 2007 se evaluaron prospectivamente 75 pacientes, realizándose 96 exámenes en total. Hubo un franco predominio masculino (2/3 del grupo), y la edad promedio de los pacientes evaluados fue de 65,6 $\pm 23,3$ años (rango: 12-99 años). $B$ examen se realizó utilizando un nasofaringolaringoscopio flexibley administrando alimento (papilla y líquido espeso) teñido con colorante azul natural. En ficha especialmente diseñada se registraron los hallazgos del examen, en especial la presencia de penetración laríngea y de aspiración traqueal del alimento.

Resultados: Los diagnósticos neurológicos fueron los más habituales (54,7\%), predominando la patología cerebrovascular, el traumatismo encéfalo-craneano y lapatología neurológica degenerativa Sólo el 17,8\% de los pacientes presentaron una evaluación completamente normal. La alteración más frecuente fue la presencia de residuos alimentarios posdeglución (79,3\%); lapenetración laríngeay aspiración traqueal se demostraron en el 46, 1\% y el 27,3\% de las evaluaciones, respectivamente. Tanto la presencia de traqueostomía como la retención de secreciones hipofaríngeas se asociaron, en forma significativa, al hallazgo de aspiración alimentaria No se logró demostrar una asociación entre el diagnóstico de base del paciente y la severidad de la aspiración. No hubo complicaciones durante la realización del examen.

Conclusiones: La evaluación fibroscópica de la deglución es un examen sensible, validado, seguro y confiable en el estudio de la disfagia orofaríngea. Entre sus ventajas destaca la portabilidad y la ausencia de exposición a radiación. Se enfatiza la importancia de contar con alimentos de consistencia estandarizada, una ficha única de registro y criterios comunes en la interpretación de los diferentes hallazgos del examen.

Palabras clave: Disfagia orofaríngea, evaluación fibroscópica, deglución, ÆES.

\section{ABSTRACT}

Introduction. Cropharyngeal dysphagia is a frequent pathological condition, specially in the elderly. It is associated to several diseases (mainly neurological and traumatic) and it

\footnotetext{
Médico Otorrinolaringólogo, Clínica Las Condes.

Interno de Medicina, Universidad de Chile.

Interno de Medicina, Universidad Diego Portales.

Fonoaudióloga, Clínica Las Condes.
} 
carries a risk of pneumonia and aspiration. Fibroscopic evaluation of swallowing allows the study of the physiology of swallowing, the assessment of aspiration risk, and enables counseling as to the safest way to feed the patient.

Aim. To review the experience in fibrocopic evaluation of swallowing in our institution

Material and method: Seventy five patients were prospectively evaluated between April 2006 and July 2007, for a total of 96 fibroscopies. Two-thirds of the evaluated patients were males, with an average age of 65.6 23.3 years (ranging from 12 to 99 years). Examination was made by means of a flexible nasopharyngoscope while administering food (purée and thick liquid) stained with a natural blue dye. Findings were registered in a custom-designed data sheet, emphasizing the presence of laryngeal penetration and tracheal aspiration of food.

Results. The more frequent diagnoses were neurological (54.7\%), with a predominance of cerebrovascular pathology, cranial trauma and degenerative neurological pathology. Only $17.8 \%$ of evaluations were considered completely normal. The most frequent alteration was the presence of post-swallowing food residues (79.3\%); laryngeal penetration and tracheal aspiration were demonstrated in $46.1 \%$ and $27.3 \%$ of evaluations, respectively. Both the presence of tracheostomy and of hypopharyngeal secretion retention were significantly associated to food residues presence. An association between the patient's diagnosis and the aspiration severity failed to be demonstrated. No complications were reported during examination.

Conclusion. Swallowing evaluation by fibroscopy is a validated, safe and sensitive examination technique for the evaluation of oropharyngeal dysphagia It has the advantage of easy-to-carry equipment and no need to radiation exposure. The need to use standard consistency food, a common datasheet to record findings and uniform criteria for the interpretation of the findings is stressed.

Key words. Oropharyngeal dysphagia, fibroscopic evaluation, swallowing, ÆES.

\section{INTRODUCCIÓN}

Disfagia es la sensación de dificultad en el avance de la comida desde la boca al estómago; una definición más completa es la de Johnson: "Trastorno para tragar alimentos sólidos, semisólidos y/o líquidos por una deficiencia en cualquiera de las cuatro etapas de la deglución y que puede desencadenar neumonía, desnutrición, deshidratación y obstrucción de la vía aérea"1. La disfagia es una condición patológica frecuente, presente en el 6$9 \%$ de la población general. Su prevalencia aumenta en forma considerable con la edad, afectando al $16 \%-22 \%$ de los mayores de 50 años y al $60 \%$ de los pacientes añosos que viven en casas de reposo. La gravedad de la disfagia radica en su asociación con neumonías aspirativas, las que tienen un $40 \%$ de mortalidad, representando la cuarta causa de muerte en adultos mayores ${ }^{2}$.

La incidencia de la disfagia está aumentando, debido a una serie de causas. El sostenido envejecimiento de la población es un fenómeno que está ocurriendo a nivel mundial y Chile no es la excepción: actualmente, los mayores de 65 años representan el $7,9 \%$ de la población chilena ${ }^{3}$. La disfagia en pacientes de edad avanzada, así como las comorbilidades asociadas (enfermedad pulmonar obstructiva crónica, cardiopatías, desnutrición, diabetes), elevan en 6 veces el riesgo de desarrollar una neumonía ${ }^{4}$. Por otro lado, algunas patologías de alta complejidad y de manejo intrahospitalario que se asocian a trastornos de la deglución han elevado su incidencia y mejorado sus tasas de sobrevida, dando origen a una población de pacientes con disfagia secuelar. Un ejemplo es el accidente cerebro vascular (AVE), cuadro de incidencia progresiva con la edad, que puede presentar disfagia hasta en el $80 \%$ de los casos 5 . También se ha descrito una alta frecuencia de disfagia y aspiración en pacientes de unidades de cuidados intensivos que han estado intubados por más de 48 horas $^{6}$.

La disfagia, al alterar los mecanismos de protección de la vía aérea durante la deglución, au- 
menta el riesgo de aspiración. Puede ocurrir que el bolo alimenticio caiga prematuramente desde la boca hacia la faringe (antes de que la laringe esté preparada, es decir, elevada y con aducción cordal), 0 bien que queden residuos alimentarios posdeglución en hipo y orofaringe. Cuando esta descoordinación orofaríngea se asocia a patrones respiratorios alterados (respiración rápida 0 incoordinada), el riesgo de aspiración aumenta ${ }^{7}$. Sin embargo, no todo evento de aspiración de contenido faríngeo lleva a una neumonía; en muchos casos se produce una limpieza eficaz del aspirado, lo que explicaría la prevalencia de hasta un $50 \%$ de aspiración silenciosa en sujetos sanos durante el sueño ${ }^{8}$. La aspiración es causa necesaria, pero no suficiente, para provocar una neumopatía aspirativa. Entre los factores predisponentes para que un episodio de aspiración desencadene una neumonía están las características del aspirado (volumen, $\mathrm{pH}$, carga bacteriana) y las características del huésped (eficacia de la tos, clearance mucociliar, status inmunológico $)^{5}$.

Asumiendo que las neumonías aspirativas pueden ser prevenidas mediante la detección precoz y manejo adecuado de la disfagia, se han desarrollado una serie de métodos para el estudio de la deglución. La evaluación de la disfagia orofaríngea debe tener como objetivos: 1) identificar causas estructurales, 2) evaluar la integridad funcional de la deglución orofaríngea, 3) evaluar el riesgo de aspiración y, por ende, de desarrollar una neumonía aspirativa, y 4) determinar si el patrón de disfagia es tratable. Entre las técnicas de evaluación de la disfagia orofaríngea, destacan la evaluación clínica (en inglés, bedside evaluation) con 0 sin oximetría de pulso, la videofluoroscopía y la evaluación fibroscópica de la deglución ( inglés, por Fberoptic Endoscopic Evaluation of Swallowing). La evaluación clínica, si bien es económica y disponible, tiene una validez limitada y su interpretación es muy variable. La videofluoroscopía, en cambio, es un examen objetivo y altamente sensible, sin embargo su aplicación se ve limitada por una serie de problemas: exposición a radiación, necesidad de desplazar al paciente a radiología y no es representativo del contexto cotidiano en que se alimenta el paciente ${ }^{8}$. Desde su primer reporte, en el año 1988, la evaluación fibroscópica de la deglución ha demostrado ser una técnica objetiva, confiable y sensible para el diagnóstico de la disfagia orofaríngea. Es un examen versátil, portátil y seguro, generalmente bien tolerado, que permite evaluar e implementar maniobras compensatorias para permitir una deglución segura ${ }^{9}$. Para su realización, se requiere de un nasofaringolaringoscopio flexible, disponible en prácticamente la totalidad de los Servicios de Otorrinolaringología. La duración del examen es de entre 10 y 20 minutos $^{10}$.

El objetivo del presente estudio es exponer la experiencia del Departamento de Otorrinolaringología de Clínica Las Condes en la evaluación fibroscópica de la deglución. Se analizará la técnica empleada, la población estudiada y los resultados obtenidos en estas evaluaciones.

\section{MATERIAL Y MÉTODO}

\section{Grupo de estudio}

Entre abril del año 2006 y julio del año 2007 se evaluaron prospectivamente 75 pacientes, realizándose 96 exámenes en total. Estos pacientes fueron derivados por sus médicos tratantes por ser portadores (o tener sospecha) de una disfagia orofaríngea. En el grupo de estudio había un franco predominio masculino, con 50 individuos $(66,7 \%$ del total). La edad promedio de los pacientes evaluados fue de 65,6 $\pm 23,3$ años, con un rango que se extendía entre los 12 y 99 años. Estas evaluaciones fueron realizadas a solicitud del equipo tratante del paciente, cuando se sospechaba de algún grado de disfagia $y / 0$ cuando se quería evaluar la posibilidad de realimentar a un paciente.

\section{Técnica de evaluación}

En la mayoría de los pacientes se realizó la nasofaringolaringoscopía previa aplicación de oximetazolina tópica, pero sin utilizar anestesia. La endoscopía, inicialmente, debe evaluar los siguientes aspectos: anatomía faringo-laríngea, manejo de saliva y secreciones, efectividad de la tos, movilidad del velo del paladar, contractibilidad faríngea, elevación laríngea y movilidad cordal (aducción y abducción). Toda lesión y/o alteración anatómica debe ser consignada en detalle. La retención de 
saliva es un parámetro muy relevante, ya que se ha demostrado que se correlaciona con el riesgo de aspirar, probablemente secundario a un déficit sensitivo en la hipofaringe. Se consigna la severidad de la retención de secreciones con una escala modificada de Donzelli1 ${ }^{11}$, que va desde grado 1 (escasas secreciones ocupan menos del $10 \%$ de senos piriformes y valléculas) hasta grado 4 (franca aspiración de secreciones).

A continuación, se le administra el alimento al paciente. En esta etapa del examen, es muy importante el posicionamiento del nasofibroscopio. Previo a la deglución, el endoscopio debe ubicarse entre el velo del paladar y la epiglotis, para evaluar la llegada del bolo a la faringe; en esta posición se logra visualizar toda la laringe e hipofaringe. La etapa faríngea de la deglución, desde el punto fibroscópico, es una etapa ciega: la imagen se "blanquea" debido a la elevación del paladar blando y a la contracción de los constrictores faríngeos. Después de la deglución, el nasofibroscopio se avanza hacia el vestíbulo laríngeo, permitiéndonos evaluar la existencia de penetración y/o aspiración de alimento a la vía aérea ${ }^{10}$. Es recomendable continuar observando en esta posición durante un lapso de tiempo, para determinar la presencia de regurgitación posdeglución ( $p$. ej. en pacientes portadores de un divertículo de Zenker).

Las características del alimento a ser aportado son críticas al momento de interpretar el estudio de deglución. Es por esto que, en conjunto con nutricionistas y fonoaudiólogas de nuestra institución, se estandarizó una bandeja de alimentos para evaluación de deglución. Esta bandeja incluye dos consistencias de alimento: papilla y líquido espeso, que se elaboran a partir de colado de frutas y de néctar de frutas, respectivamente. En ambas preparaciones se utiliza una cantidad pre-establecida de espesante Thick \& Easy ${ }^{\circledR}$ (que no requiere cocción), además de un colorante azul vegetal. Se administra primero la papilla, continuando luego con el líquido espeso. Para ambas viscosidades se administran cantidades progresivas, a partir de $1 / 4$ de cucharada. En caso de evidenciarse a la nasofibroscopía un episodio de aspiración, se realiza un segundo intento; si el paciente aspira nuevamente, se lo considera "reprobado" para esa consistencia y volumen. Es muy importante realizar evaluaciones repetidas para ambas consisten- cias, dado que la coordinación de la deglución puede alterarse por la fatiga.

Para cada consistencia se registra la existencia y el grado (leve-moderado-severo) de:

1) Derrame posterior (spillage en inglés), corresponde a la permanencia del bolo alimentario en hipofaringe (senos piriformes) por más de 2 segundos antes del inicio de la etapa faríngea de la deglución.

2) Residuos: Persistencia de alimento en las paredes faríngeas, senos piriformes o valléculas después de producida la deglución.

3) Penetración laríngea: Entrada de alimento al vestíbulo laríngeo, por sobre el nivel de las cuerdas vocales verdaderas.

4) Aspiración: El alimento desciende más allá del nivel de las cuerdas vocales verdaderas, hacia la tráquea.

5) Reflujo: La regurgitación de alimento desde el esófago de regreso a la laringo-faringe.

El grado de severidad de estos hallazgos la determina el evaluador en forma subjetiva, según una estimación de la cantidad de alimento que penetra 0 es aspirado a la vía aérea 0 del tiempo que permanece el alimento en hipofaringe previo a la deglución (derrame) o posterior a ésta (residuos).

Debemos determinar en qué momento de la deglución ocurre la aspiración (si es que se produce), de modo de comprender la fisiopatología de la disfagia en un paciente dado y poder planificar su rehabilitación. Cuando la aspiración ocurre en la etapa pre-deglución, ésta se puede deber a la existencia de derrame oral posterior 0 a una demora en el inicio de deglución. La aspiración durante la deglución se produce por un cierre laríngeo enlentecido $y / 0$ incompleto. Si hay aspiración posdeglución, ésta puede ser secundaria a la presencia de residuos alimentarios en la hipofaringe.

Es de gran importancia contar con la asistencia del fonoaudiólogo del equipo de deglución en las evaluaciones endoscópicas, para determinar en conjunto los volúmenes, consistencias y maniobras deglutorias a incorporar en el paciente, así como para planificar el tratamiento a seguir.

Todos los hallazgos de este examen se consignan en el Informe sobre Evaluación Fibroscópica de la Deglución (ver anexo 1). 
Anexo 1. Informe de Evaluación Fibroscópica de la Deglución.

\section{EVALUACIÓN FIBROSCÓPICA DE LA DEGLUCIÓN}

\section{Identificación}

Nombre:

Edad: Habltacion: $N^{2}$ Ficha:

Examen soliditado por:

Diagnastico de base:

Anamnesis deglución:

Condiclones del examen:

Fecha:

Estado de conciencia

Clósido

Desorientado variable

Soperoso

Posicibn:

Traquestomia:

Semisentado $\left(45^{5}\right)$ en cana

(1)

No Mecicamentos utilzados:

\section{Estudio anátomico-funcional de faringo-laringe}

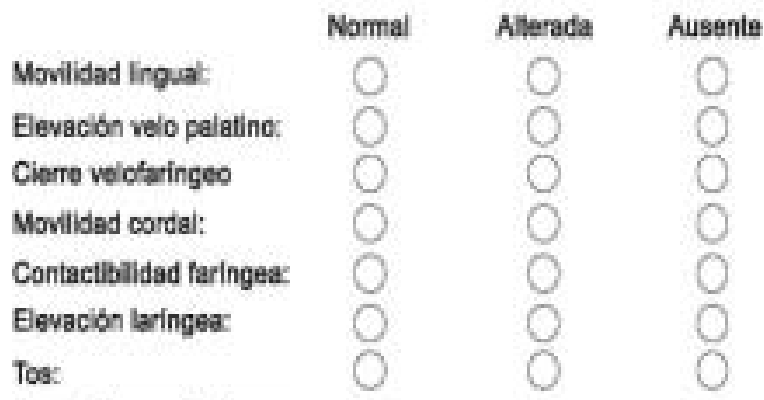

Anomalias anatbmicas:

Acumulacion de secreciones:

Grado 1 (escasa secrecianes ocupan <10\% de senos pirlformesivalieculas)

Grado 2 (abundartes secreciones apumuladas en senos pirilormes/valéculas)

Grado 3 (ingreso de aecreciones a vestibulo laringeo, sin aspiracion)

Grado 4 (aspiración de socraciones) 


\section{Estudio dinámico de la degluclón}

Alimentos stilixades:

Pasilin Liquide con espesants

Otro

Volumen tolerade:

Q 1/4 cosharads

Q $1 / 2$ cucharsts

Cucharsis complets

Sarbos do tazo

o bembila

Administración de alimento:

Q El mieme paciente $O$ Anistida

Hallargos endoscopicos:

\begin{tabular}{|l|l|l|l|l|l|}
\hline & Ausente & Leve & Moderado & Severo & Aute-limpieza \\
\hline Derrame (apWagor" & & & & & \\
\hline Residuos (peoing)" & & & & & \\
\hline Penetracion laringes & & & & \\
\hline Aspiracion & & & & \\
\hline Reflujo & & & & \\
\hline
\end{tabular}

- Derrame: Bolo alimenticio entra a hipofaringe $>2$ segundes antes de que se inicie degluoion

Comentarios adicionales:

\section{Conclusiones}

Diagnbatica:

Recomendaciones

Evaluacion / manojo por fonouvdiologla: $\bigcirc$ si $\bigcirc$ No Apto para alimentación por via oral:

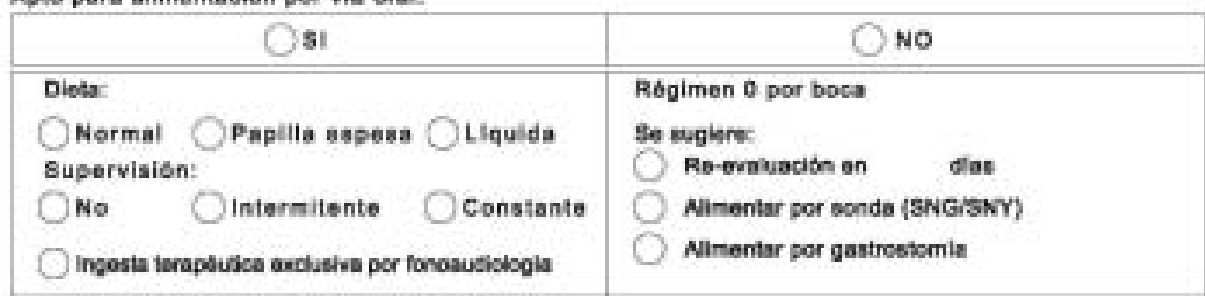

Observaciones:

Examinador: Dr. 


\section{Análisis estadístico}

Los datos recolectados en los informes de los pacientes fueron analizados mediante estadísticas descriptivas. Se estudió la presencia de asociaciones entre diferentes variables, mediante la aplicación de la herramienta que correspondiera en cada caso: test de MannWhitney (asociación entre traqueostomía y grado de retención de secreciones/severidad de aspiración), test exacto de Fisher (asociación entre aspiración y presencia de traqueostomía) y Chi cuadrado (asociación entre aspiración y compromiso de conciencia/diagnósticos de base/retención de secreciones). Se consideraron significativas todas aquellas asociaciones con un valor estimado de $p$ menor a 0,05. Los cálculos estadísticos se realizaron utilizando los programas Excel ${ }^{\circledR}$ y Primer of biostatistics ${ }^{\circledR}$ (versión 3,0).

\section{RESULTADOS}

En la población estudiada ( $n=75)$, los diagnósticos de base correspondieron en su mayoría a patología neurológica, con 41 casos $(54,7 \%)$. Dentro de este grupo, hubo un franco predominio de la patología cerebrovascular (16 casos, $21,3 \%$ del total), seguido del traumatismo encéfalo craneano (TEC) y de la patología neurológica degenerativa. Otro grupo diagnóstico importante corresponde a pacientes con neumonías recurrentes $\mathrm{y} / 0$ prolongadas ( 9 casos), en las cuales se sospechaba una etiología aspirativa. Como tercer grupo diagnóstico impor- tante se puede mencionar a los pacientes con patología grave, ya sea cardiovascular 0 abdominal, que hayan requerido monitorización intensiva e intubación orotraqueal (Tabla 1).

Si se analizan los diagnósticos por grupos etarios, se aprecia un predominio de los TEC en pacientes jóvenes, mientras que la patología cerebrovascular y neurológica degenerativa se concentran en pacientes mayores de 65 años. De forma similar, los pacientes que eran evaluados por neumopatías recurrentes o por la sola sospecha de disfagia (sin otro diagnóstico especificado), eran en su mayoría adultos mayores (Figura 1).

Sobre las condiciones del examen, éste fue realizado en posición sentada (espalda en $90^{\circ}$ ) en apenas el $39 \%$ de los casos, mientras que en los demás pacientes $(61 \%)$ sólo se pudo obtener una posición de "semisentado" en 45․ El estado de conciencia fue descrito como lúcido en el 57,6\% de los pacientes al momento del examen, encontrándose algún grado de compromiso de conciencia (desorientación o franco sopor) en el 42,4\% restante. Veinte coma seis por ciento de los pacientes estaban traqueostomizados cuando fueron evaluados.

En el $25,5 \%$ de los pacientes evaluados se apreció una retención anormal de secreciones y/o saliva en hipofaringe, la cual era francamente aspirada en el $6,4 \%$ de los casos. Se demostró una correlación entre la presencia de traqueostomía y una mayor retención de secreciones hipofaríngeas $(p=0,038)$. En este sentido, cabe señalar que todos los pacientes

Tabla 1. Diagnósticos de base de pacientes sometidos a evaluación de deglución

\begin{tabular}{|lcc|}
\hline Grupos diagnósticos & Casos & Porcentaje \\
\hline Neurológico (total) & \multicolumn{2}{c|}{54,7} \\
$\quad$ Neurológico vascular & 16 & 21,3 \\
Neurológico traumático (TEC) & 7 & 9,3 \\
Neurológico degenerativo & 7 & 9,3 \\
Neurológico misceláneo & 11 & 14,7 \\
Neumopatía recurrente & 9 & 12 \\
Cardiovascular & 5 & 6,7 \\
Patología abdominal & 4 & 5,3 \\
Patología vía aérea & 6 & 8 \\
Oncológico & 3 & 4 \\
Disfagia, sin otra especificación & 7 & 9,3 \\
Total & 75 & 100 \\
\hline
\end{tabular}




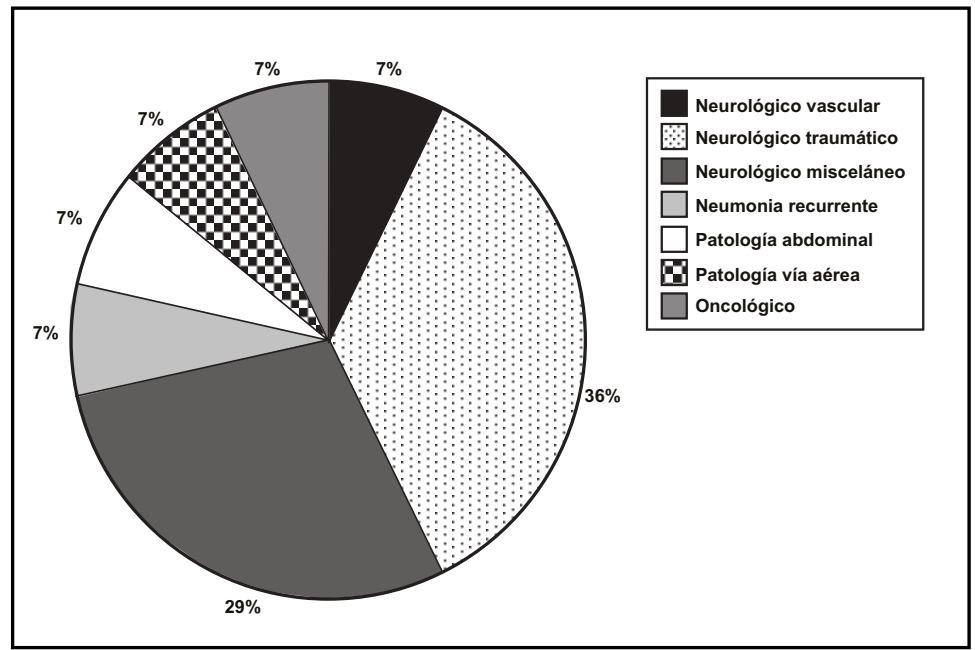

Figura 1. Diagnósticos de base según grupos etarios.

Figura 1A. Diagnósticos de base en pacientes menores de 40 años.

Figura 1B. Diagnósticos de base en pacientes con edades entre 40 y 65 años.

Figura 1C. Diagnósticos de base en pacientes mayores de 65 años.

con aspiración espontánea de saliva estaban traqueostomizados, y en el $50 \%$ de los casos su diagnóstico de base correspondía a un TEC.

El $97,7 \%$ de los pacientes requirió que se le administrara el alimento en la boca, siendo incapaces de alimentarse por sí mismos al momento del examen. En el estudio dinámico de la deglución, sólo el 17,8\% de los pacientes presentaron una evaluación completamente normal. La alteración más frecuente fue presentar algún grado de residuos alimentarios posdeglución en la hipofaringe (79,3\%), mientras que la presencia de penetración laríngea y aspiración fue demostrada en el $46,1 \%$ y el $27,3 \%$ de las evaluaciones, respectivamente (Tabla 2).

La presencia de traqueostomía se asoció significativamente a una mayor incidencia $(p=0,014)$ y severidad $(p=0,037)$ de aspiración durante el examen. Se demostró también una significativa asociación $(p<0,001)$ entre retención de secreciones hipofaríngeas y aspiración alimentaria (Tabla 3).

Si bien se observó una mayor incidencia de aspiración en pacientes con compromiso de conciencia (desorientados y/o soporosos), esta asociación no fue estadísticamente significativa $(p=0,062)$. Finalmente,

Tabla 2. Hallazgos en estudio dinámico de la deglución

\begin{tabular}{|lcccccc|}
\hline & $\mathrm{n}^{*}$ & Ausente & Leve & Moderado & Severo & $\%$ alteración \\
\hline Derrame & 88 & 58 & 14 & 12 & 4 & 34,1 \\
Residuos & 87 & 18 & 37 & 25 & 7 & 79,3 \\
Penetración laríngea & 89 & 48 & 25 & 15 & 1 & 46,1 \\
Aspiración & 88 & 64 & 17 & 5 & 2 & 27,3 \\
Reflujo & 84 & 70 & 8 & 4 & 2 & 16,7 \\
\hline
\end{tabular}

$\mathrm{n}$ *: Número de casos en que estaba registrada la información. 
Tabla 3. Asociación entre retención de secreciones y aspiración alimentaria

\begin{tabular}{|lccccc|}
\hline \multirow{2}{*}{ Retención de secreciones } & \multicolumn{4}{c|}{ Severidad de aspiración alimentaria } \\
& Ausente & Leve & Moderada & Severa & $\%$ aspiración \\
\hline Grado 1 & 45 & 5 & 0 & 1 & $11,8 \%$ \\
Grado 2 & 3 & 3 & 2 & 0 & $62,5 \%$ \\
Grado 3 & 2 & 1 & 1 & 0 & $50 \%$ \\
Grado 4 & 0 & 3 & 0 & 1 & $100 \%$ \\
\hline
\end{tabular}

no fue posible establecer una asociación significativa entre el diagnóstico de base del paciente y la severidad de la aspiración. No obstante lo anterior, los casos de aspiración moderada y severa se dieron en los pacientes con diagnósticos de patología cerebrovascular, insuficiencia cardíaca, patología abdominal complicada y patología de vía aérea alta (absceso periamigdalino). Por otro lado, los pacientes con diagnósticos oncológicos (linfoma avanzado, cáncer prostático) y diagnósticos neurológicos "misceláneos" (disfunción pseudobulbar, neuropatías motoras, encefalopatía, tetraplejia, patología neuroquirúrgica) no presentaron ningún grado de aspiración en nuestra serie (Tabla 4).

En la presente serie, no se reportaron complicaciones en relación a la realización del examen.

\section{DISCUSIÓN}

La evaluación fibroscópica de la deglución (0 FES constituye una excelente herramienta en el estudio del paciente portador de una disfagia orofaríngea. Con su realización, se puede determinar la presencia de anomalías anatómicas en la vía aerodigestiva superior y la fisiología de la etapa faríngea de la deglución. Al determinar el riesgo de

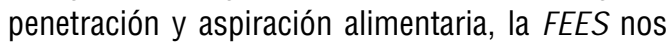
ayuda a decidir qué pacientes se puede realimentar y a quiénes debe mantenerse en régimen 0 por boca (alimentación enteral por sonda nasogástrica, nasoyeyunal o gastrostomía). La evaluación fibroscópica de la deglución posee además una serie de ventajas respecto de otros exámenes como la videofluoroscopía, al ser un examen portátil, reproducible y que no implica exposición a radiación.

La validez de la $\mathbb{E}$ Squedó demostrada en los estudios de Langmore, quien estimó que este examen tenía $88 \%$ de sensibilidad y $90 \%$ de concordancia con la videofluoroscopía respecto de la presencia de aspiración ${ }^{9}$. Se ha cuestionado incluso que la videofluoroscopía (VF) siga siendo el

Tabla 4. Diagnósticos de base y severidad de aspiración

\begin{tabular}{|c|c|c|c|c|}
\hline \multirow[b]{2}{*}{ Grupos diagnósticos } & \multicolumn{4}{|c|}{ Severidad de aspiración } \\
\hline & Sin aspiración & Leve & Moderada & Severa \\
\hline Neurológico total & 29 & 5 & 1 & 1 \\
\hline Neurológico vascular & 13 & 0 & 1 & 1 \\
\hline Neurológico traumático (TEC) & 4 & 3 & 0 & 0 \\
\hline Neurológico degenerativo & 3 & 2 & 0 & 0 \\
\hline Neurológico misceláneo & 9 & 0 & 0 & 0 \\
\hline Neumopatía recurrente & 6 & 2 & 0 & 0 \\
\hline Cardiovascular & 1 & 2 & 1 & 0 \\
\hline Patología abdominal & 3 & 0 & 0 & 1 \\
\hline Patología vía aérea & 3 & 2 & 1 & 0 \\
\hline Oncológico & 2 & 0 & 0 & 0 \\
\hline Disfagia, sin otra especificación & 6 & 1 & 0 & 0 \\
\hline
\end{tabular}


gold standard del estudio de la disfagia orofaríngea: Wu y cols. ${ }^{12}$ encontraron una superioridad de la $\mathbb{E E S}$ respecto de la VF en la detección tanto de penetración laríngea como de aspiración traqueal, al evaluar 28 pacientes con ambos exámenes.

El principal objetivo de los estudios de la deglución orofaríngea es la prevención de neumopatías aspirativas. En este sentido, la efectividad de la $\rightleftarrows E S$ fue sugerida por un estudio realizado por Spiegel ${ }^{13}$, quien reportó la ausencia de neumonías aspirativas en pacientes derivados de hogares de ancianos, una vez que se introdujo la evaluación fibroscópica de la deglución. En forma similar, Ajemian y cols. ${ }^{14}$ no tuvieron episodios de disfagia "clínicamente significativa" ni neumonías en un grupo de 51 pacientes con intubación prolongada, recientemente extubados y evaluados por FES. Probablemente la evidencia más sólida en esta línea es un estudio randomizado realizado por Aviv $^{15}$, en el que evaluó la incidencia de neumonía en pacientes disfágicos, comparando a un grupo evaluado con videofluoroscopía con otro grupo evaluado con la técnica de inglés, por Fiberoptic Endoscopic Evaluation of Swallowing with Sensory Testing) complementa la evaluación fibroscópica de la deglución descrita por Langmore con una evaluación sensitiva, determinando el umbral del reflejo aductor laríngeo a través el uso de pulsos de aire. En su estudio, Aviv describió una menor incidencia de neumonía en el grupo evaluada por \#ESST; esta diferencia alcanzó significación estadística en el grupo de pacientes con AVE.

La $\mathbb{E}$ S ha demostrado ser un estudio muy seguro $^{10}$. Entre sus principales complicaciones se ha mencionado la epistaxis $(0,6 \%)$, las reacciones vaso-vagales $(0,06 \%)$ y el laringoespasmo $(0,03 \%)$. En el presente estudio, no se reportó ninguna complicación durante la realización de la FES.

La correcta interpretación de la evaluación fibroscópica de la deglución requiere de criterios comunes entre los examinadores y de una herramienta estable en el tiempo (es decir, que no cambie de un caso a otro). Con esta finalidad es que se ha diseñado una ficha de registro única y se ha estandarizado la preparación de los alimentos de la bandeja de deglución. En ciertas situaciones puede ser deseable contar con múltiples consistencias de alimentos (p. ej. durante la rehabilitación de una disfagia orofaríngea). Sin embargo, para fines diagnósticos nos ha parecido suficiente el evaluar a la mayoría de los pacientes con dos consistencias: papilla y líquido espeso. Intencionalmente no se han incluido líquidos (p. ej. agua), puesto que la deglución de éstos es más dificultosa y se asocia a mayor riesgo de aspiración en pacientes con disfagia posAVE'16. Tampoco utilizamos jalea, ya que, en nuestra experiencia, constituye un alimento que sobreestima la penetración a la vía aérea, al tener aspecto sólido pero fluir como líquido. El alimento es teñido con un colorante azul vegetal; no utilizamos azul de metileno, puesto que se han reportado reacciones adversas ocasionales con el uso de este colorante, incluido el shock anafiláctico ${ }^{17}$. Por otro lado, los pacientes suelen referir cambios en el sabor de los alimentos al usar el azul de metileno.

En un intento por aumentar la objetividad de la \#ES, se han diseñado algunas escalas para evaluar la severidad del trastorno de la deglución. La escala PAS (penetration-aspiration scale) fue diseñada por Rosenbek para calificar la severidad de la disfagia en pacientes evaluados con videofluoroscopía. Colodny adaptó y validó esta escala en la evaluación fibroscópica de la deglución ${ }^{18}$. En la $\mathbb{E}$ S, esta escala tiene una buena correlación intra e interevaluador, distinguiendo bien entre penetración y aspiración. Sin embargo, algunos autores sugieren que la penetración y la aspiración son problemas diferentes, que debieran ser evaluados separadamente y no como parte de una misma escala. Al no existir aún un consenso respecto de una escala de evaluación de disfagia, en nuestra ficha hemos optado por describir los diferentes aspectos de la deglución faríngea sin entregar una "puntuación final".

Una de las ventajas de la evaluación fibroscópica de la deglución es que no requiere demasiada colaboración del paciente: a diferencia de la videofluoroscopía, suele ser suficiente con que el paciente obedezca algunas órdenes simples. No obstante lo anterior, la $\mathbb{E} E$ debe realizarse en las mejores condiciones posibles. Idealmente, el paciente debe estar lúcido, bien sentado (espalda en $90^{\circ}$ ) y haber sido decanulado (si es que fue sometido a una traqueostomía). En nuestra serie, la presencia de traqueostomía se asoció significativamente a un peor resultado en la evalua- 
ción endoscópica de la deglución. Sin embargo, en la práctica clínica diaria se suelen pedir evaluaciones de deglución a pacientes con algún grado de compromiso de conciencia $(42,4 \%)$, que apenas pueden semisentarse (61\%) y que aún no han sido decanulados (20,6\%). En estos casos, cuando el examen resulta alterado, debe sugerirse una reevaluación cuando el paciente haya sido decanulado y se encuentre en mejores condiciones desde el punto de vista neurológico, esperando encontrar entonces una mejor fisiología deglutoria y así aliviar las restricciones de alimentación que se puedan haber indicado.

En los pacientes que se puedan realimentar, el examen permite la implementación de técnicas compensatorias (p. ej. postura cefálica, deglución en apnea) para minimizar el riesgo de aspiración traqueal. Estas maniobras se indican de acuerdo a los hallazgos del examen, teniendo en cuenta la fisiopatología de la disfagia en el paciente en particular, y su utilidad debe demostrarse durante el examen ${ }^{10}$. En este sentido, la $\rightleftarrows E S$ puede servir como biofeedback (retroalimentación), si al paciente se le muestra la imagen endoscópica en pantalla y se le explican las alteraciones que deben ser corregidas. La evaluación fibroscópica de la deglución también permite establecer recomendaciones dietéticas: consistencias alimentarias (eventual uso de espesantes), tamaño del bolo que el paciente puede manejar en forma segura y volumen total a administrar. Se deben indicar las recomendaciones dietéticas más seguras pero menos restrictivas (ni muy conservador, ni muy liberal), buscando así una aceptable calidad de vida para el paciente ${ }^{10}$.

En aquellos pacientes que presentan un alto riesgo de aspiración, no se les debe permitir la alimentación por vía oral. Alternativas en este caso son las sondas de alimentación enteral y la gastrostomía. Sin embargo, no existe evidencia concluyente que demuestre que las sondas enterales reducen el riesgo de neumonía aspirativa en pacientes con disfagia ${ }^{19}$. Por lo tanto, en todo paciente consciente, es recomendable hacer el máximo esfuerzo de que sea alimentado por la boca, ya sea en forma total 0 en forma parcial y progresiva (ingesta de tipo terapéutica). El trabajo dedicado del equipo de deglución (otorrinolaringólogo y fonoaudiólogo) logra un buen resultado en la mayoría de los casos. En nuestra opinión, se debiera reservar la alimentación enteral mediante sondas o gastrostomía para aquellos pacientes con compromiso de conciencia, pacientes con tos extremadamente incómoda durante la alimentación y pacientes con historia de neumonía aspirativa recurrente a pesar de los mayores esfuerzos de rehabilitación.

\section{CONCLUSIONES}

La evaluación fibroscópica de la deglución ( es una técnica sensible, validada, segura y confiable en el estudio de la disfagia faríngea. Entre sus ventajas destaca la portabilidad y la ausencia de exposición a radiación. Permite analizar la fisiología de la deglución, determinar el riesgo de aspiración y, en conjunto con el fonoaudiólogo, indicar la dieta y maniobras compensatorias que permitan una deglución segura.

\section{BIBLIOGRAFÍA}

1. Johnson ER, McKenzie SW, Sievers A. Aspiration pneumonia in stroke. Arch Phys Med Rehabil 1993; 74: 973-6.

2. LIND CD. Dysphagia: evaluation and treatment. Gastroenterol Cin NAm 2003; 32: 553-75.

3. Aguilera M, TorRejón M. Chile hacia el 2050: Proyecciones de población. Instituto Nacional de Estadísticas 2005; 1: 5-29.

4. MariK PE, Kaplan D. Aspiration pneumonia and dysphagia in the elderly. Chest 2003; 124: 328-36.

5. PerRY L, Love CP. Screening for dysphagia and aspiration in acute stroke: a systematic review. Dysphagia 2001; 16: 7-18.

6. Barquist E, Brown M, Cohn S, Lundy D, Jackowski J. Postextubation fiberoptic endoscopic evaluation of swallowing after prolonged endotracheal intubation: A randomized, prospective trial. Orit Care Med 2001; 29: 1710-3.

7. Morton R, Minford J, Ellis R, Pinnington L. Aspiration with dysphagia: the interaction between oropharyngeal and respiratory impairments. Dysphagia 2002; 17: 192-6.

8. Ramsey D, Smithard D, Kalra L. Silent aspiration: what do we know? Dysphagia2005; 20: 218-25. 
9. Langmore SE, Schatz K, Olson N. Endoscopic and videofluoroscopic evaluations of swallowing and aspiration. Ann Otol Rhinol Laryngol 1991; 100: 678-81.

10. Hiss SG, Postma GN. Fiberoptic Endoscopic Evaluation of Swallowing. Laryngoscope 2003; 113: 1386-93.

11. Donzelli J, Brady S, Wesling M, Craney M. Predictive value of accumulated oropharyngeal secretions for aspiration during video nasal endoscopic evaluation of the swallow. Ann Otol Rhinol Laryngol 2003; 112: 469-75.

12. Wu CH, Hsiao TY, Chen JC, Chang YC, Lee Sy. Evaluation of swallowing safety with fiberoptic endoscope: comparison with videofluoroscopic technique. Laryngoscope 1997; 107: 396-401.

13. Doggett DL, Tappe KA, Mitchel MD et al. Prevention of pneumonia in elderly stroke patients by systematic diagnosis and treatment of dysphagia: an evidence-based comprehensive analysis of the literature. Dysphagia2001; 16: 279-95.

14. Ajemian MS, Nirmul GB, Anderson Mt, Zirlen DM, KWASNIK EM. Routine fiberoptic endoscopic evaluation of swallowing following prolonged intubation: implications for management. Arch Surg 2001; 136: 434-7.

15. Aviv JE. Prospective, randomized outcome study of endoscopy versus modified barium swallow in patients with dysphagia. Laryngoscope2000; 110: 563-74.

16. Trapl M, Enderle P, Nowotny M et al. Dysphagia bedside screening for acute-stroke patients: the gugging swallowing screen. Stroke 2007; 38: 1-7.

17. Dewachter P, Mourton-Faivre C, Tréchot P, Lleu JC, MeRTES PM. Severe anaphylactic shock with methylene blue instillation. Anesth Analg 2005; 101: 149-50.

18. Colodny N. Interjudje and intrajudge reliabilities in fiberoptic endoscopic evaluation of

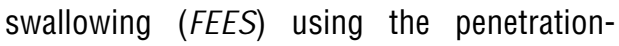
aspiration scale: a replication study. Dysphagia 2002; 17: 308-15.

19. Finucane TE, Bynum JPW. Use of tube feeding to prevent aspiration pneumonia. Lancet 1996; 348: 1421-4. 